\title{
Author Correction: Per2 induction limits lymphoid-biased haematopoietic stem cells and lymphopoiesis in the context of DNA damage and ageing
}

Jianwei Wang, Yohei Morita, Bing Han, Silke Niemann, Bettina Löffler and K. Lenhard Rudolph

Correction to: Nature Cell Biology https://doi.org/10.1038/ncb3342, published online 18 April 2016.

In the version of this Article originally published, the authors mistakenly used the same images for the Fig. $2 \mathrm{~d}$ Per ${ }^{-/-}$upper Merge, DAPI and p-RPA2 (Ser33), and Fig. 3e upper 2 months, WT Per2, DAPI and Merge panels. The correct images from these experiments, and their correctly sized scale bars, are shown below. In addition, the full dataset of representative images for Figs. $2 \mathrm{~d}$ and $3 \mathrm{e}$, and the numerical source data of the corresponding quantifications of Figs. 2c and 3f, have been uploaded to Figshare (https://doi.org/10.6084/ m9.figshare.c.4353791.v1). In addition, the Methods section did not provide sufficient detail on how immunoblot experiments were conducted. Western blots were repeated three times using the antibodies stated in the Methods. PER2 blots were obtained using the PER2 antibody (Millipore, AB2202); except in the PER2 blots in the Repeat 1 experiments corresponding to Figs. 3g and 3k, where the PER2 antibody from BD Transduction (611138) was used. Repeats 1 and 2 were imaged using ECL and LI-COR methods, and Repeat 3 only used the LI-COR system. The images presented in the paper were from Repeat 3 and were stored electronically and as printouts, however the electronic versions were cropped as depicted in Supplementary Fig. 6. Repeat 1 images were stored electronically and Repeat 2 images only as printouts. In addition - with the exception of Fig. 2g (ATM), $3 g$ (PER2, Repeat 1A), 3k (PER2, PUMA, p21, BAX, BCL2) and Supplementary Fig. 1a of Repeat 1, in which actin immunoblots were loading controls processed from the same membranes used to detect the target proteins - in all other cases the actin blots were sample processing controls that were run in parallel using the same lysates and loading concentrations that were used for the detection of the target proteins. The electronic images from Repeat 1 and the printout images of Repeats 2 and 3 have been uploaded to Figshare (https://doi.org/10.6084/m9.figshare.c.4353791.v1). Finally, there is an error in the protein size label of the p-CHK1 blot of Fig. $2 \mathrm{f}$ and the corresponding blot in Supplementary Fig. 6, which should read '55KD' instead of '50KD'. The corrected panels are shown below.
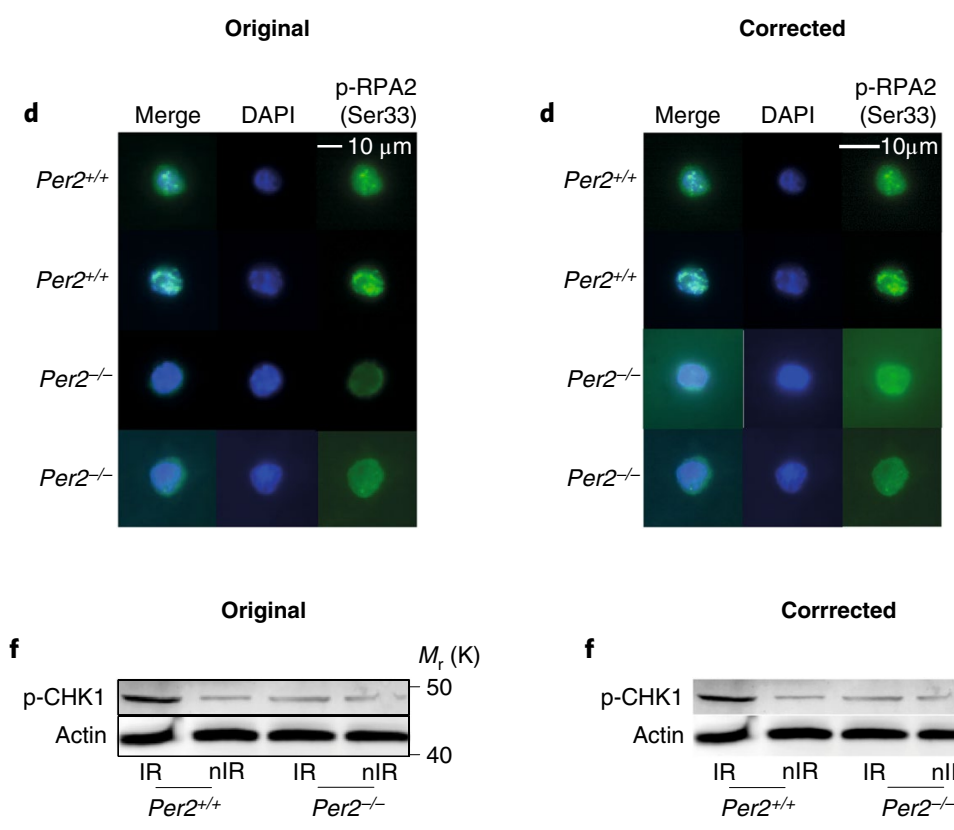

Fig. 2 | Original and corrected.

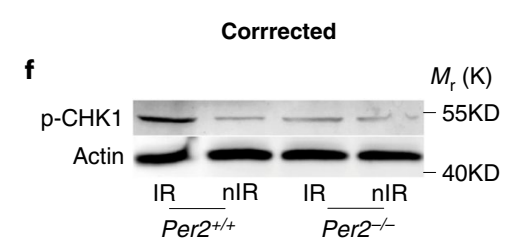

\footnotetext{
Fig. 2 Original and corrected.
} 


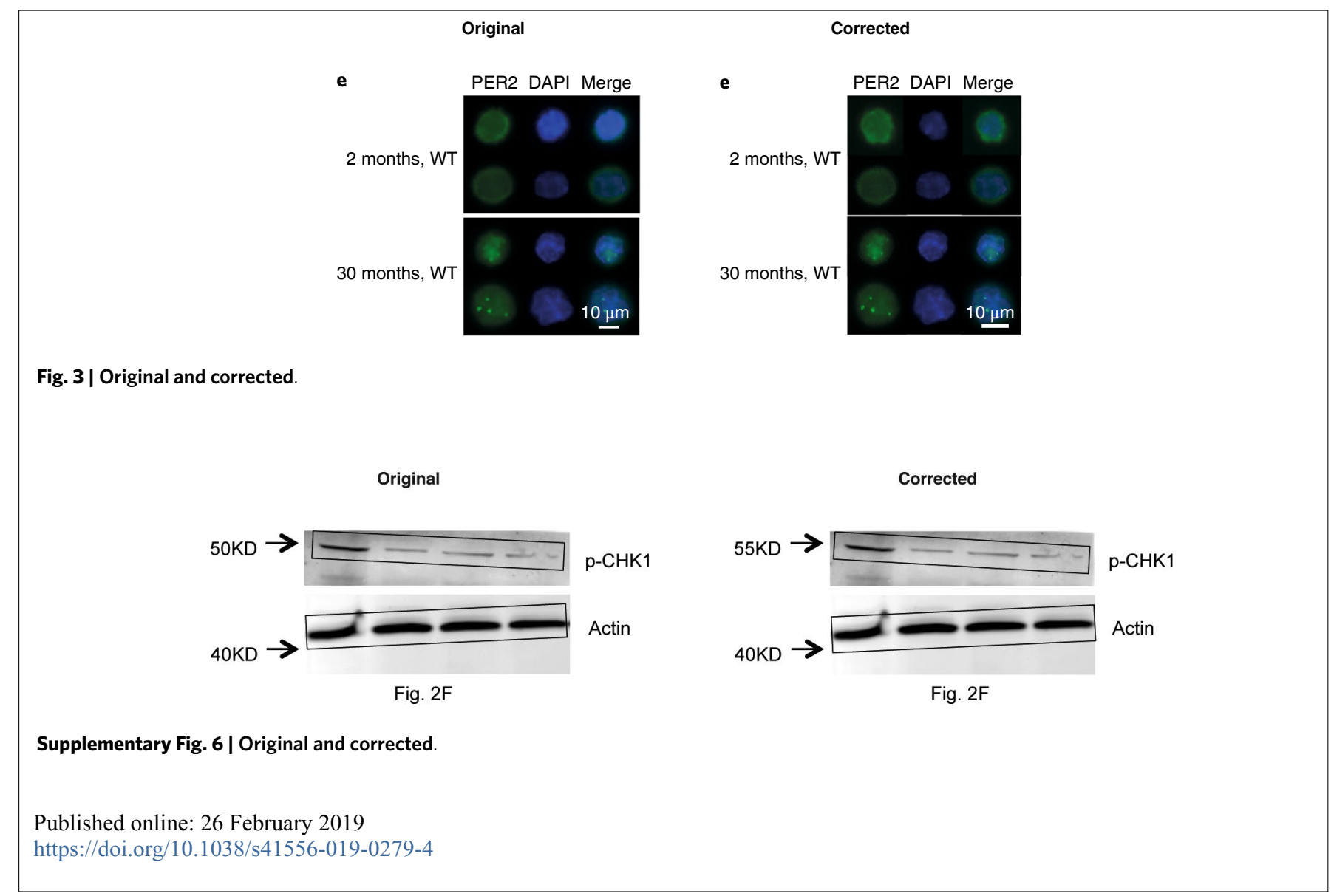

\section{Author Correction: Recapitulating endocrine cell clustering in culture promotes maturation of human stem-cell-derived $\beta$ cells}

Gopika G. Nair, Jennifer S. Liu, Holger A. Russ, Stella Tran, Michael S. Saxton, Richard Chen, Charity Juang, Mei-lan Li, Vinh Q. Nguyen, Simone Giacometti, Sapna Puri, Yuan Xing, Yong Wang, Gregory L. Szot, Jose Oberholzer, Anil Bhushan and Matthias Hebrok (D)

Correction to: https://doi.org/10.1038/s41556-018-0271-4, published online 1 February 2019

In the version of this article originally published, the Gene Expression Omnibus (GEO) accession number listed in the data availability section was incorrectly given as GSE10979 instead of GSE109795. The sentence should read "RNA-seq data that support the findings of this study have been deposited in the Gene Expression Omnibus (GEO) under accession code GSE109795," and the code should link to https://www.ncbi.nlm.nih.gov/geo/query/acc.cgi?acc=GSE109795. The error has been corrected in the HTML and PDF versions of the paper. 